\title{
DKVF: A Framework for Rapid Prototyping and Evaluating Distributed Key-Value Stores *
}

\author{
Mohammad Roohitavaf \\ Computer Science and Engineering Department, \\ Michigan State University \\ East Lansing, MI, USA \\ roohitav@cse.msu.edu
}

\author{
Sandeep Kulkarni \\ Computer Science and Engineering Department, \\ Michigan State University \\ East Lansing, MI, USA \\ sandeep@cse.msu.edu
}

\begin{abstract}
We present our framework DKVF that enables one to quickly prototype and evaluate new consistency protocols for key-value stores. DKVF is designed based on the separation of concerns in creating distributed data stores. This separation of concerns allows the designers of consistency protocols to only focus on the highlevel consistency protocols which gives them the opportunity to quickly deploy a consistency protocol and evaluate its performance. Moreover, the loose coupling of the different components allows us to easily change different components (e.g. storage engine) of an implementation. We demonstrate DKVF by implementing four existing protocols -eventual consistency, COPS, GentleRain and CausalSpartan- with it. The implementation of these protocols was very convenient with DKVF, as it only required to write a piece of code for the consistency component that is very close to the pseudocode of the original papers. Hence, it was possible to achieve this in just 1-2 days per protocol. DKVF also comes with a toolset that facilitates running clusters and performing experiments. Tutorial video: https://www.youtube.com/watch?v=MFJQzsJkwfc\&list= PLErtSVEHsnBJvoQQI6iqGn61oNrUVVuST
\end{abstract}

\section{CCS CONCEPTS}

- Computer systems organization $\rightarrow$ Cloud computing;

\section{KEYWORDS}

Distributed Data Stores, Key-value Stores, Framework, Prototyping, YCSB, Geo-replication

ACM Reference Format:

Mohammad Roohitavaf and Sandeep Kulkarni. 2018. DKVF: A Framework for Rapid Prototyping and Evaluating Distributed Key-Value Stores . In Proceedings of the 201833 rd ACM/IEEE International Conference on Automated Software Engineering (ASE '18), September 3-7, 2018, Montpellier, France. ACM, New York, NY, USA, 4 pages. https://doi.org/10.1145/3238147.3240476

*This work is supported in part by NSF XPS 1533802.

Permission to make digital or hard copies of all or part of this work for personal or classroom use is granted without fee provided that copies are not made or distributed for profit or commercial advantage and that copies bear this notice and the full citation on the first page. Copyrights for components of this work owned by others than ACM must be honored. Abstracting with credit is permitted. To copy otherwise, or republish, to post on servers or to redistribute to lists, requires prior specific permission and/or a fee. Request permissions from permissions@acm.org.

ASE '18, September 3-7, 2018, Montpellier, France

(C) 2018 Association for Computing Machinery.

ACM ISBN 978-1-4503-5937-5/18/09...\$15.00

https://doi.org/10.1145/3238147.3240476

\section{INTRODUCTION}

With the huge amount of data and very high query throughput produced by a large number of users across the world, storing data in a single machine does not work for any major business. Thus, we have to distribute the data across several machines. When we distribute our data, an important challenge is the consistency between different copies (i.e., replicas) of the data. There is an inherent trade-off between consistency and availability/performance [5]. Different levels of consistency come with different levels of availability/performance overhead. Even to achieve a certain level of consistency, two different protocols may have different levels of overhead.

In general, this suggests that developers need to develop new protocols to improve performance, provide higher levels of consistency, reduce communication requirements, reduce storage requirements, and so on. When the developers intuitively identify a new approach to design such a protocol, the natural question that arises is how to evaluate the new protocol by comparing it with different existing protocols. Distributed data stores are complex systems which makes an accurate analytical performance evaluation infeasible for them. A more practical option is experimental performance evaluation via benchmarking a prototype running the protocol.

In this paper, we introduce Distributed Key-Value Framework (DKVF) [3] that allows protocol designers to quickly create prototypes running their protocols to see how they work in practice. Using separation of concerns, DKVF allows researchers to only focus on their high-level protocol, and rely on DKVF for all the lower-level tasks. This approach greatly expedites implementing a prototype for a given protocol compared with creating a prototype from scratch. For instance, consider the GentleRain protocol proposed in [4]. The server-side of this protocol is only 31 lines of pseudocode provided in Algorithm 2 of [4]. However, to have a prototype running this protocol, we need to write hundreds of lines of code to handle lower-level tasks that are independent of the protocol. Our goal is to provide a framework that helps researchers to create their prototypes by writing codes that are very close to the pseudocodes that they publish in their research papers. We believe this framework together with a toolset that helps us to run experiments can significantly save time in implementing and benchmarking new protocols.

The rest of this paper is organized as follows: Section 2 discusses the separation of concerns in designing distributed data stores, Section 3 reviews the implementation using DKVF. Section 4 introduces the tools that comes with DKVF. Section 5 provides some of the experimental results. Finally, Section 6 concludes the paper and provides future work. 


\section{SEPARATION OF CONCERNS}

DKVF is designed based on the separation of concerns of implementing a distributed key-value store. Figure 1 shows three different components of a distributed data store. The storage component is responsible for the storage and retrieval of the data in a single machine. It does not interact with either the clients or other servers. It only serves the read/write requests from the consistency component. The communication component is responsible for server-server and client-server communications. The communication component can provide different guarantees. For instance, it may provide total order, per-channel FIFO, or no ordering guarantee; be reliable or unreliable; be synchronous or asynchronous, and so on. The consistency component is responsible for defining replication strategy and consistency protocol that guarantees clients always observe a consistent view of the data. Different levels of consistency is defined for replicated data stores. The highest level of consistency is called strong consistency that provides the illusion that there is only a single copy of the data. On the other end, eventual consistency only guarantees that all replicas finally converge to the same data if we stop writing new values. In between, we have different levels of intermediate consistency models such as causal consistency [7]. A consistency protocol requires a set of requirements for the storage and communication components. However, the consistency protocol should not be involved in providing these requirements. This loose coupling lets us easily change different components in both design and implementation of a distributed data store.

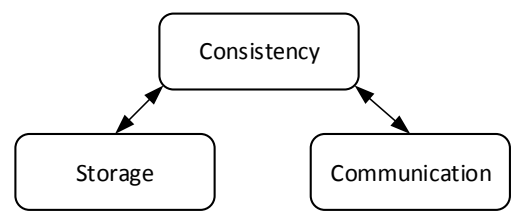

Figure 1: Components of a distributed data store.

\section{IMPLEMENTATION USING DKVF}

To implement a protocol using DKVF, the protocol designer needs to provide three sub-components of the consistency component of Figure 1.

\subsection{Metadata Description}

For a consistency protocol, in addition to actual data consisting of the key-value pairs, we will likely need to store some metadata with each record. For example, we may need to store a timestamp with each version, or we may need to store the ID of the replica where the version has been written. Each protocol requires its own metadata. DKVF relies on Google Protocol Buffers [6] for marshaling/unmarshaling data for storage and transmission. The protocol designer needs to write a . proto text file describing the metadata. This file also describes the structure of the messages sent in the system.

\subsection{Server-side}

To implement the server-side of a protocol, the protocol designer needs to write a class that extends the abstract class DKVFServer.
DKVF follows an event-driven approach to define a protocol. Specifically, we can define a protocol as a set of event handlers. The two main event handlers that will be called by the framework are handleServerMessage and handleClientMessage of DKVFServer class. Inside these two main event handlers, the protocol designer can call detailed event handlers for different events. While we are processing server or client messages in handleServerMessage and handleClientMessage, we may need to send messages to other servers, or send client responses. DKVF provides methods to reliably send/receive messages to/from clients and servers. We also need to store/retrieve data from the storage engine that can be done via that DKVF storage interface. Any data storage engine can be used with DKVF. To use a storage engine, we have to provide DKVF driver for it by implementing the DKVF storage interface. DKVF comes with a driver for Oracle Berkeley-DB [9].

\subsection{Client-side}

To implement the client-side of a protocol, we need to extend the client part of the framework. Specifically, we need to write a class that extends class DKVFClient. When we extend DKVFClient, we have to implement two abstract methods put and get that are the basic PUT and GET operations of a key-value store. These methods are operations that the protocol designer needs to provide for the application developer. The application developer later can use these methods to use the data store. The protocol designer can also add more complex operations for its implementation, but these two methods are required for any implementation.

\section{TOOLSET}

In this section we reviews tools that come with the framework.

\subsection{Cluster Manager}

Cluster Manager is a command line application to facilitate managing clusters running key-value stores created with DKVF. Cluster Manager also enables us to monitor the servers. For instance, we can see if servers have properly started and connected to each other, how much are the network latencies, or how many clients are connected to each server. Cluster Manager also helps us to test and debug our key-value store. Specifically, after running a cluster we can connect to any server in the cluster and run commands on the servers.

It also helps us to run distributed YCSB [2] experiments. YCSB is a tool for evaluating the performance of key-value or cloud serving stores [2]. To use YCSB, we need to write a YCSB driver that lets YCSB client class use our key-value store. DKVF comes with its YCSB driver. Thus, any key-value store using DKVF has its YCSB driver ready. Also using Cluster Manager tool, we can benchmark our key-value store without directly setting up YCSB; we only need to define our desired workload, and Cluster Manager takes cares of the rest. It runs all clients and obtains the results. Cluster Manager provides an interactive environment with a query language that lets us aggregate the results.

\subsection{Cluster Designer}

Although Cluster Manager is a convenient tool that can significantly reduce time and headache of debugging and benchmarking our 


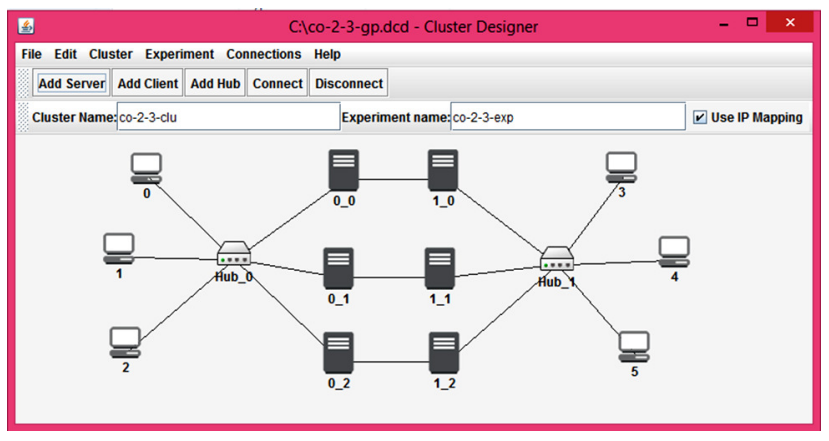

Figure 2: The graphical interface of Cluster Designer

protocol, writing cluster and experiment descriptor files can be a tedious and error-prone task for larger clusters. To solve this issue, we provide Cluster Designer tool. Cluster Designer is a graphical tool that allows us to define our cluster and experiments visually. The tool provides an area where we can add servers and clients. We can connect servers and clients by lines to specify network connections. When we have several components that need to be all connected to each other, we can use hubs to avoid connecting them one-by-one. Figure 2 shows the interface of Cluster Designer. In this network, we have 6 servers and 6 clients. We will talk about this network in more details in 5 .

\section{EXPERIMENTAL RESULTS}

In this section, we present some of the results that we obtained from implementing and evaluating three causal consistency protocols namely COPS [7] ${ }^{1}$, GentleRain [4], and CausalSpartan [10] using DKVF. We also implemented eventual consistency for comparison. Due to lack of space, we cannot provide the sketch of these protocols. The reader can refer to the original papers to learn more about these protocols. We assume the reader is familiar with these protocols in the rest of this section.

We consider a replicated and partitioned data store shown in Figure 2. The data store consists of two replicas. Each replica consists of three partitions. Replica 0 includes partitions $0 \_0,0 \_1$, and $0 \_2$. Replica 1, on the other hand, consists of partitions 1_0, 1_1, and 1_2. We assume full replication, i.e., each replica has a copy of the entire key space. The key space inside each replica is partitioned among servers. In Figure 2, we have connected servers inside each replica together with a hub. Partitions are also connected to their peers in the other replica. For servers, we use AWS [1] m3 .medium instances with the following specification: 1 vCPUs, $2.5 \mathrm{GHz}$, Intel Xeon E5-2670v2, 3.75 GiB memory, 1 x 4 (GB) SSD Storage Capacity.

Connected to each replica, we have a set of clients. We allocate three client machines to run clients. We run 30 threads of YCSB clients on each client machine. All causal consistency protocols that we study here assume locality of traffic, i.e., clients always access one replica. Thus, clients are connected to only one replica as shown Figure 2. We run clients on c3. large machines with the following specification: 2 vCPUs, $2.8 \mathrm{GHz}$, Intel Xeon E5-2680v2,

\footnotetext{
${ }^{1}$ We have implemented a simplified version of COPS without garbage collection.
}

3.75 GiB memory, 2 x 16 (GB) SSD Storage Capacity. We have used more powerful machines for clients to better utilize our servers.

\subsection{The Effect of Workload on Performance}

The workload of different applications has different characteristics. Some workloads are write-heavy, others like those in data analytics are read-heavy. In this section, we want to study how the characteristics of our workload affect the performance of different consistency protocols. In all experiment, we set the size of the values written by clients to 64 bytes.

Figure 3a shows how GET:PUT proportion affects the throughput. As we move from the left side of the plot to its right side, the workload nature changes from write-heavy to read-heavy. The throughputs of all protocol increase as the proportion of GET operations increases. This results confirm previous studies [4, 7], and are expected, as GET operations are lighter than PUT. As expected, eventual consistency has the highest throughput. COPS, on the other hand, has the lowest throughput. This results confirm results published in [4], and is due to the overhead of dependency check messages that partitions send to each other to make sure causal dependencies of an update in other partitions are visible [4, 7].

Figure 3b shows how GET:PUT proportion affects the response time of PUT operations. In all protocols, the response time of PUT operations decreases as we move to read-heavier workloads. This is due to the less load on servers for read-heavier workloads. The eventual consistency has the shortest response time thanks to its minimal metadata. CausalSpartan has more metadata than GentleRain resulting in higher PUT response time. COPS has the highest response time because of its dependency check messages and its explicit dependency tracking approach. Like other protocols, the trend of PUT response time for COPS is decreasing as we move toward read-heavier workloads that can be explained by less load on the machines. However, for 0.05:0.95, the PUT response time increases. This increase can be understood by considering the dependency tracking mechanism of COPS. At point 0.05:0.95, clients read many keys before writing a key. That results in longer dependency lists which make PUT messages heavier to transmit and process. Note that we have implemented a basic version of COPS protocol without client metadata garbage collection. COPS authors suggest a garbage collection mechanism to cope with this problem [7].

Figure $3 c$ shows how GET:PUT proportion affects the response time of GET operations. Like the case of PUT operations, the response time of GET operations also decreases, as we move towards read-heavier workloads. It is interesting that GentleRain and CausalSpartan have a lower response time for GET operations comparing to the eventual consistency for write-heavy workloads. This can be explained by the synchronization that occurs between threads in GentleRain and CausalSpartan. Specifically, there is a contention between threads while performing PUT operations in GentleRain/CausalSpartan. This contention occurs for obtaining a lock that we used to guarantee updates with smaller timestamps are replicated to other nodes before updates with higher timestamps. This increases the PUT response time that results in a lower overall throughput of GentleRain/CausalSpartan for write-heavy workloads. While threads serving PUT operations are waiting for 
synchronization, the server can handle GET operations. On the other hand, in the eventual consistency, there is no competition between PUT operations. Thus, there are more active threads serving PUT operations leading to higher competition over CPU that finally results in higher GET response time comparing to GentleRain/CausalSpartan. Note that this happens for write-heavy workloads with low GET proportion. Therefore, the eventual consistency still has the highest overall throughout in all cases (See Figure 3a).

\subsection{The Effect of Query Amplification}

In this section, we study the effect of query amplification that causes a single end user request translate to several internal operations. In this section, we only consider one replica consisting of three partitions. We consider a workload that purely consists of amplified insert operations. Each amplified insert consists of several internal PUT operations. The number of internal PUT operations is defined by the amplification factor.

Figure $3 \mathrm{~d}$ shows the effect of amplification factor on the client request throughput. Note that this throughput represents the number of client macro operations (not individual PUT operations) that are served in one second. As the amplification factor increases, the throughput of all protocol decreases which is expected, as requests with higher amplification factor include more internal operations which mean more job to do for each request. The eventual consistency has the highest throughput. The pure-write workload is an ideal write scenario for COPS, as dependency lists have at most one entry. Thus, the throughput of COPS is the highest after eventual consistency for this scenario. GentleRain has the lowest throughput. That is due to the delay that GentleRain imposes on PUT operations in case of clock skew between servers. Note that we synchronized the physical clocks of the system with NTP [8], but the effect of clock skew still shows up in the results. These results confirm previous results presented in [10]. CausalSpartan has higher throughput than GentleRain, as CausalSpartan eliminates the need for the delay before PUT operations by utilizing HLCs instead of physical clocks[10]. Figure 3e shows the request response time for different protocols. Again, because of delays that GentelRain forces on PUT operations, request response time has the highest value for GentleRain.

\section{CONCLUSION}

In this paper, we introduced DKVF which is a framework for rapid prototyping and benchmarking distributed key-value stores. It streamlines the evaluation of the performance of consistency protocols for distributed key-value stores. To show the effectiveness of our framework, we implemented four consistency protocols using DKVF. Thanks to the convenience of DKVF, we were able to implement each of these protocols in less than 2 days. We were able to implement CausalSpartan and GentleRain with significantly less effort than our previous implementations without DKVF.

The toolset that comes with the framework helps protocol designers to easily evaluate their prototype. Using these tools we can easily run and manage clusters of machines running our protocol. We can also run clients to benchmark the performance of our key-value store. DKVF relies on YCSB for benchmarking.

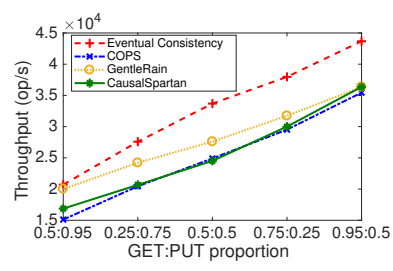

(a) Throughput

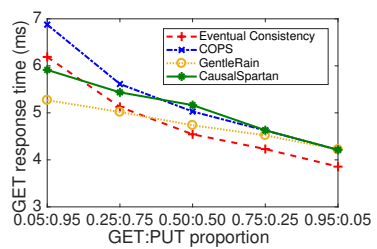

(c) GET Response Time

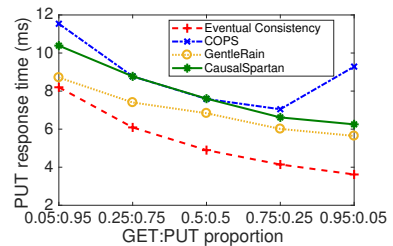

(b) PUT Response Time

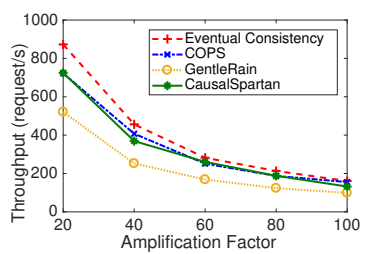

(d) Request Throughput

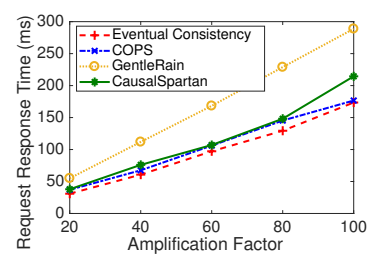

(e) Request Response Time

Figure 3: Some of results obtained from DKVF

We can use any storage systems as the storage engine for the key-value stores that we develop with DKVF. This enables protocol designers to flexibility change their storage engine. To use a given storage system with DKVF, we need to write a driver for it that enables DKVF to interact with it. DKVF comes with a driver for Berkeley-DB. Writing drivers for other storage systems is part of the future work.

\section{REFERENCES}

[1] Amazon. 2018. AWS. (2018). https://aws.amazon.com

[2] Brian F Cooper, Adam Silberstein, Erwin Tam, Raghu Ramakrishnan, and Russell Sears. 2010. Benchmarking cloud serving systems with YCSB. In Proceedings of the 1st ACM symposium on Cloud computing. ACM, 143-154.

[3] DKVF. 2018. GitHub repository. (2018). https://github.com/roohitavaf/DKVF

[4] Jiaqing Du, Călin Iorgulescu, Amitabha Roy, and Willy Zwaenepoel. 2014. GentleRain: Cheap and Scalable Causal Consistency with Physical Clocks. In Proceedings of the ACM Symposium on Cloud Computing (SOCC '14). New York, NY, USA, Article 4, 4:1-4:13 pages.

[5] Seth Gilbert and Nancy Lynch. 2002. Brewer's Conjecture and the Feasibility of Consistent, Available, Partition-tolerant Web Services. SIGACT News 33, 2 (June 2002), 51-59.

[6] Google. 2018. Protocol Buffers. (2018). https://developers.google.com/ protocol-buffers/

[7] Wyatt Lloyd, Michael J. Freedman, Michael Kaminsky, and David G. Andersen. 2011. Don'T Settle for Eventual: Scalable Causal Consistency for Wide-area Storage with COPS. In Proceedings of the Twenty-Third ACM Symposium on Operating Systems Principles (SOSP '11). New York, NY, USA, 401-416.

[8] NTP. 2018. Network Time Protocol. (2018). http://www.ntp.org/

[9] Oracle. 2018. BerkeleyDB. (2018). http://www.oracle.com/technetwork/database/ database-technologies/berkeleydb/overview/index.html

[10] Mohammad Roohitavaf, Murat Demirbas, and Sandeep Kulkarni. 2017. CausalSpartan: Causal Consistency for Distributed Data Stores using Hybrid Logical Clocks. In Reliable Distributed Systems (SRDS), 2017 IEEE 36th Symposium on. IEEE, 184-193. 